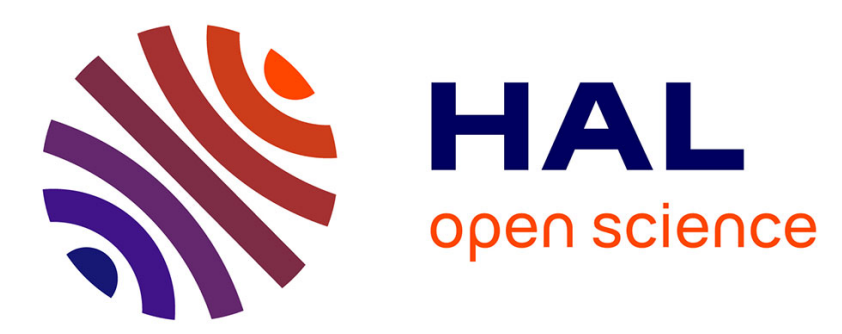

\title{
Les lecteurs parlent: Les plus grands arbres du monde (toujours à propos des Sequoias) - Industrie papetière -
} La forêt: Usine à bois

- [.]revue Forestière Française, Rédaction

\section{- To cite this version:}

- [.]revue Forestière Française, Rédaction. Les lecteurs parlent: Les plus grands arbres du monde (toujours à propos des Sequoias) - Industrie papetière - La forêt: Usine à bois. 1957, pp.314. $10.4267 / 2042 / 27300$. hal-03535802

\section{HAL Id: hal-03535802 https://hal.science/hal-03535802}

Submitted on 19 Jan 2022

HAL is a multi-disciplinary open access archive for the deposit and dissemination of scientific research documents, whether they are published or not. The documents may come from teaching and research institutions in France or abroad, or from public or private research centers.
L'archive ouverte pluridisciplinaire HAL, est destinée au dépôt et à la diffusion de documents scientifiques de niveau recherche, publiés ou non, émanant des établissements d'enseignement et de recherche français ou étrangers, des laboratoires publics ou privés. 


\section{LES LECTEURS PARLENT}

\section{Les plus grands arbres du monde (toujours à propos des Sequoias)}

L'auteur de l'article: " $A$ propos des sequoias de Californie », paru dans le numéro de janvier I957, a reçu du Père de Tarade, dendrologue éminent, connu des lecteurs de la Revue par l'article ( les principales essences forestières du Liban », paru en juin I950, une lettre dont il nous paraît intéressant de donner quelques extraits à nos lecteurs:

« J'ai lu votre dernier article de la Revue forestière sur les Sequoias. Je possède la petite brouchure: «The giant sequoias of California 》 que vous citez et qui est remarquablement présentée. M. Emmanuel FrITZ, professeur à la School of Forestry de Berkeley, qui est chargé, je crois, des $\mathrm{Na}$ tional Parks de Redwoods, m'avait aussi envoyé plusieurs brochures intéressantes du même genre, éditées par la « Save the Redwoods League 》, en échange de renseignements sur quelques arbres de cette espèce ( 3 en tout) que je connaissais au Liban.

Pour les dimensions maxima mondiales, relevées sur des arbres, la référence généralement citée est: TiemanN (H.-D.), « What are the largest trees in the world? 》, Journal of Forestry (1935), 33, p. 903-I5, où, avec les Sequoias, sont cités quelques arbres de dimensions analogues, arbres de la flore malaise (Koompossia excelsa) de 84 mètres (Bornéo et Péninsule malaise) et quelques Kauri (Agathis alba et australis) qui atteindraient 70 à 75 mètres, avec, pour le dernier, des diamètres assez proches des sequoias. Ces chiffres sont cités dans P.-W Richards, «The Tropical Rain Forest 》, que j'ai ici.

On trouve, comme vous dites, beaucoup de chiffres énormes sur les dimensions (surtout sur les hauteurs plus difficiles à vérifier) même dans des livres sérieux (130 mètres pour un eucalyptus dans BüsGEN et MUNCH) et une critique est nécessaire. Le Gouvernement australien avait, je crois, offert vers I920-25 une énorme prime à qui indiquerait et mesurerait un eucalyptus ayant au moins $120 \mathrm{~m}$. Elle n'a jamais été réclamée et les plus beaux arbres signalés atteignaient 90 à $95 \mathrm{~m} . \gg$

\section{Industrie papetière}

\section{Nous recevons d'un lecteur la lettre suivante:}

Monsieur le Rédacteur en chef,

Auriez-vous l'amabilité de me dire s'il est exact que l'Assemblée Nationale a été saisie par un groupe de parlementaires d'une proposition de résolution tendant à inviter le gouvernement à promouvoir en France une industrie papetière à base de matière première nationale qui serait le sorgho.

Cette plante bien que rustique serait cultivée dans les terres à blé irriguées du midi de la France.

Les pouvoirs publics ont-ils renoncé à faciliter les investissements pour 
améliorer l'utilisation et la production de nos matières premières déjà nationales depuis longtemps telles que le bois, et la paille dont l'excédent : est exporté et transformé à l'étranger notamment en Hollande pour nous revenir sous forme de pâte?

Veuillez...

Notre lecteur pourra en effet trouver la proposition de résolution à laquelle il fait allusion dans l'annexe du procès-verbal de la séance du 23 janiier 1957.

Nous ne pouvons malheureusement pas lui répondre en ce qui concerne les intentions du Gouvernement.

\section{La Forêt: Usine à bois}

Nous sommes heureux de faire profiter nos lecteurs des réfexions économiques très pertinentes qui viennent de nous être transmises par un abonné de marque.

Entre le bois et les matériaux dits « de remplacement 》, la lutte est depuis longtemps ouverte, dans bon nombre de domaines d'utilisation.

Non certes que les qualités, la convenance du bois soient décriées ou reniées, mais il faut constater que ce dernier tend à passer pour un matériau attardé dans 1' 《ancien 》, à faire « grand siècle 》.

$\mathrm{Au}$ cours de chaque période de crise, l'homme est amené à substituer au produit naturel à base de main-d'œuvre, un produit artificiel à base de science appliquée. La société s'équipe pour cette production de détresse née de la nécessité, la perfectionne, tant et si bien que, le calme revenu, certains " ersatz » sont à ce point réussis qu'ils subsistent pour avoir conquis les usagers par la qualité, le bon marché et surtout la présence, le tout créant l'habitude.

Il est probable que dans la tempête économique consécutive à la dernière guerre - comme il en fut des autres - le bois a quelque peu perdu les pédales.

$\mathrm{Y}$ a-t-il, à cette rupture d'équilibre, des raisons relevant du seul domaine technique qui est le nôtre à nous, forestiers?

Cette technique ne s'est-elle pas laissée prendre de vitesse par l'évolution économique? Elle qui, paisiblement, comptait, vaille que vaille avec l'éternité, è révolutions quasi-sidérales, n'est-elle pas restée à la traine d'une industrialisation lancée à une allure supersonique?

Nous avons eu Colbert, il nous reste des massifs qui font l'admiration du monde et seraient fort capables de pourvoir aux besoins d'une « marine royale » imposante. C'est cela en effet que le grand ministre avait en tête il y a quelque trois cents ans. Que la marine en bois n'ait pas duré trois siècles, ce n'est pas sa faute, du moins avait-t-il su prévoir et il était paré !

$\mathrm{Ne}$ sommes-nous plus capables (j'entends: nos grands hommes actuels) de la même simplicité de vues, de la même prévoyance, d'ailleurs terriblement raccourcie dans le temps?

Le bois doit-il toujours évoquer un tas de sciure et une pile de sciages d'importance presque comparable?

Ce que l'industrie nationale attend aujourd'hui de la forêt, c'est une masse de fibres, une masse de composés chimiques, une masse d'éléments susceptibles de transformations profondes, de manipulations complexes, voire de reconstitutions audacieuses, bref, de la masse à l'usine.

Et dès que l'on peut parler, non plus du matériau bois pris en bloc, mais de ses éléments constitutifs pris isolément, il est évident qu'un « ersatz 》: 
si parfait soit-il, a perdu la partie, le bois étant alors capable de prendre luimême les formes et les séductions d'un succédané, si j'ose le dire, de luimême.

Le problème se pose ainsi, pour la technique forestière de produire « dans le minimum de temps le maximum de matière 》, formule aujourd'hui courante qui s'agrémente, en manière de corollaire du « traitement approprié d'essences à croissance rapide $\gg$, autre formule. Ce qui manque peut-être, c'est une formule d'application permettant de répondre à un programme industriel d'ensemble.

A la cadence où va ce que l'on est convenu d'appeler le « progrès 》, prévoir à trente ans de distance est bien tout ce à quoi l'on peut prétendre si l'on veut que notre bois suive le mouvement.

Des réalisations à court terme intéressent au plus haut point la propriété privée, c'est-à-dire, la grande majorité du fonds forestier français. Un propriétaire aura toujours plaisir à réaliser, de son vivant, une fois ou même deux, le capital forestier qu'il aura eu la sagesse de se constituer ou d'épargner.

il faut que. pour le propriétaire privé au moins, la forêt cesse d'apparâitre comme une préoccupation de l'au-delà.

Par contre, il est bon que l'Etat, dans sa munificente pérennité, poursuive, au fil des siècles, la production de bois exceptionnels de grande valeur dont la réalisation entraînera d'ailleurs celle de produits accessoires ou intermédiaires parfaitement utilisables industriellement.

Le jeune forestier qui est, à présent, «Ingénieur 》 se doit de justifier l'évocation constructive de cette nouvelle appellation. Il ne peut plus se contenter d' "aider la Nature 》 dans sa lente élaboration, et de tirer parti de ce qu'elle offre spontanément; il a, bien au contraire, tout le problème à repenser pour intervenir, sans brutalité, mais avec le seul objectif d'une production rentable, intensifiée et définie pour des fins précises.

I1 lui faudra, certes, violenter un peu quelques principes consacrés par le temps, mais dépassés par une évolution d'où le facteur temps lui-même n'est pas loin d'être exclu. J'en connais pour ma part quelques-uns, ce qui n'est pas une originalité.

Enfin, le « Forestier 》 ne prendra-t-il pas, aux yeux des foules et des pouvoirs publics, une autre valeur - la vraie - si son activ̛ité se révèle directement embrayée sur le circuit industriel national, si elle en apparait nettement comme l'un des rouages essentiel, donc indispensable.

Quand l'économie nationale reconnaîtra dans la Forêt, une source de matière première, judicieusement adaptée, dans sa diversité, à ses besoins, et capable d'en suivre fidèlement l'évolution, alors, le Forestier sera viaiment l' «Ingénieur 》 privilégié d'une usine qui n'aura, pour autant, rien perdu de son attirante poésie!

E. P. 